\title{
Micro-ESR As A Laboratory Teaching Tool
}

\author{
Christine Hofstetter and James R. White
}

\author{
Active Spectrum, Inc., 1191 Chess Dr., Suite F, Foster City, CA 94404
}

\begin{abstract}
Active Spectrum's Micro-ESR spectrometer is a portable (10 kg), X-band (9.7 GHz) CW benchtop electron spin resonance spectrometer. The Micro-ESR is an ideal tool for teaching undergraduate chemistry and physics labs. This instrument enables classroom demonstrations of both simple topics such as the role of free radicals in everyday life to far less intuitive subjects including chemical bonding, electron density, spin-orbit coupling, spin-spin exchange, and forbidden transitions. The spectrometer also allows user control over many important parameters, so students can experiment with different acquisition schemes. The Micro ESR is thus ideal for an instrument lab. All data are automatically saved, and can be imported into any spreadsheet for additional processing or analysis. Offline processing software can also be used to analyze and post-process data, perform quantitative analyses, simulations and other basic functions.
\end{abstract}

Keywords: Micro-ESR, ESR, EPR, chemistry education, physics education, magnetic resonance, electron spin resonance 01.50.Pa

\section{INTRODUCTION}

Electron Spin Resonance (also called Electron Paramagnetic Resonance or EPR) is a magnetic resonance spectroscopy technique that uses the same basic principles as MRI and NMR. Both MRI and NMR interact with atomic nuclei whereas ESR interacts with unpaired electrons. Most molecules give NMR spectra even though not all nuclei can be observed by NMR because they have spin quantum number $\mathbf{I}=0$. This, however, is not true for ESR. In order for a molecule to be ESR visible, it must have at least one unpaired electron. Many compounds have only spin-paired electrons. In magnetic resonance, both NMR and ESR, it is the magnetic component of the electromagnetic radiation that interacts with the magnetic moment of the electron or nucleus. Spinpaired electrons have a net magnetic moment of zero, and therefore are ESR silent.

Electron spin resonance (ESR) was discovered in the mid-1940s. Conventional ESR spectrometers are quite large and use a large water cooled electromagnet to generate a variable magnetic field. The Micro-ESR uses a small, 0.348 Tesla, rare-earth magnet. The magnet assembly contains a low power electromagnet coil used to vary the field. The Micro-ESR is a strictly $\mathrm{CW}$ instrument with a sweep range of over 500 Gauss. The field is centered near the free electron spin gfactor. The spectrometer uses a linear voltage controlled oscillator as a microwave source, and can generate between 0.5 and $70 \mathrm{~mW}$ RF power at 9.7 GHz. The Micro-ESR uses quadrature lock-in detection, and the lock-in amplifier is internal to the system. The resonator is a cylindrical dielectric, TM011 cavity with a maximum volume of about 400 $\mu \mathrm{L}$ ( $5 \mathrm{~mm}$ diameter by $20 \mathrm{~mm}$ length). The field is homogeneous across the resonator within 0.1 Gauss. The entire Micro-ESR has a small footprint $(30.5 \mathrm{x}$ $30.5 \times 30.5 \mathrm{~cm}^{3}$ ) and a mass of only $10 \mathrm{~kg}$, so can be easily moved. The Micro-ESR requires no special installation and no special maintenance making it accessible to any undergraduate laboratory. The cost of the Micro-ESR is currently under \$40,000 USD. A block diagram of the Micro-ESR is depicted in Fig 1.

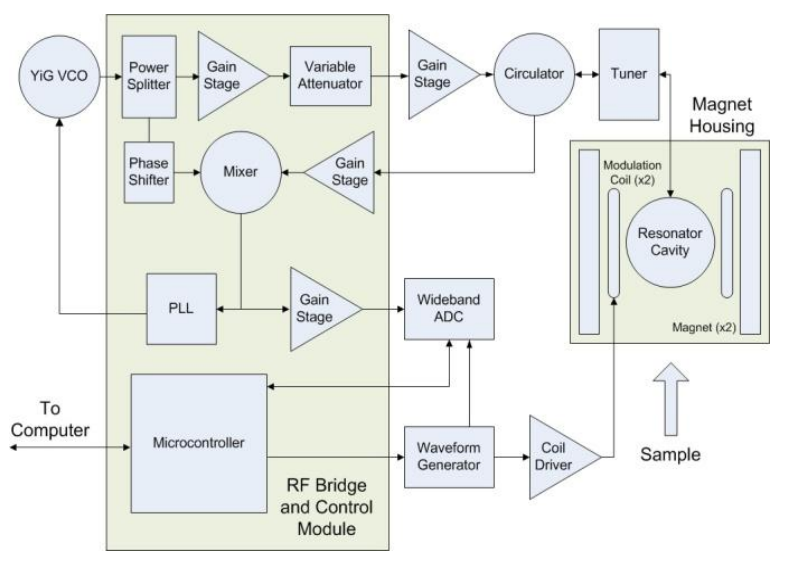

FIGURE 1. Block diagram of Micro-ESR Spectrometer.

\footnotetext{
2015 BFY Proceedings, edited by Eblen-Zayas, Behringer, and Kozminski; Peer-reviewed, doi:10.1119/bfy.2015.pr.010

Published by the American Association of Physics Teachers under a Creative Commons Attribution 3.0 license.

Further distribution must maintain attribution to the article's authors, title, proceedings citation, and DOI.
} 


\section{MAGNETIC RESONANCE IN UNDERGRADUATE EDUCATION}

Magnetic resonance is an important spectroscopic technique with which all undergraduate science students should be familiar. Although NMR is more common than ESR, ESR is still an ideal tool to give students hands-on experience with magnetic resonance. The underlying principles for both NMR and ESR are similar although the information obtained is different.

There are many topics which can be included in laboratory exercises involving ESR starting with introducing students to free radicals. Many students will be surprised to learn how many items in their everyday lives contain stable free radicals: hair, coffee, tea, and blue make-up (thiozonyl radical $^{1}$ ) to name a few compounds with stable radicals. Many transition metals have one or more unpaired electron, and can be observed by ESR in common materials.

More advanced laboratory topics dealing with less intuitive subjects like electron density ${ }^{2}$, spin-spin exchange $^{3}$, molecular dynamics ${ }^{4}$, and forbidden transitions ${ }^{5}$ are straightforward experiments for ESR. Take, for example, electron density: TEMPOL (4hydroxy-2,2,6,6-tetramethylpiperidin-1-oxyl) is a common, stable, nitroxide radical. The ESR spectrum of TEMPOL is a triplet because the unpaired electron is localized in a $\pi^{*}$ orbital on the $\mathrm{N}-\mathrm{O}$ bond and is coupled to the ${ }^{14} \mathrm{~N}$ nucleus (spin quantum number $\mathbf{I}=1$ ). There is no hyperfine splitting from either of the protons directly attached to the ring, or from any of the methyl protons. Figure 1(A) depicts the structure of the TEMPOL radical, and the corresponding ESR spectrum of three equal lines. The three lines are of equal intensity because there is an equal probability that the ${ }^{14} \mathrm{~N}$ can be seen in spin state 1,0 , or -1 . The hyperfine coupling constant for TEMPOL in water is 17.1 Gauss.

It is instructive to compare the ESR spectra of several aromatic radical anions to that of TEMPOL. Semiquinone (SQ) radical anions are relatively longlived (30-60 minutes) and are easy to generate. ${ }^{2}$ Figure 2(B and C) show the structure of two different SQ radical anions, and their respective ESR spectra. The unpaired electron in these radicals is a delocalized $\pi$ electron. Hyperfine splitting seen in the ESR spectra illustrates that the unpaired electron has density at all protons on the ring. Figure 2 (B) is a hydroquinone (1,4-Dihydroxybenzene) radical anion which has four equivalent protons. The corresponding ESR spectrum has five equally spaced lines in a 1:4:6:4:1 pattern.

Figure $2(\mathrm{C})$ is a catechol (1,2-Dihydroxybenzene) radical anion which has two sets of two equivalent protons. The ESR spectrum is a triplet of triplets.
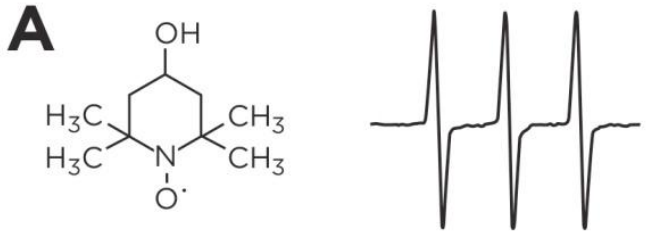<smiles>COc1ccc(O)cc1</smiles>
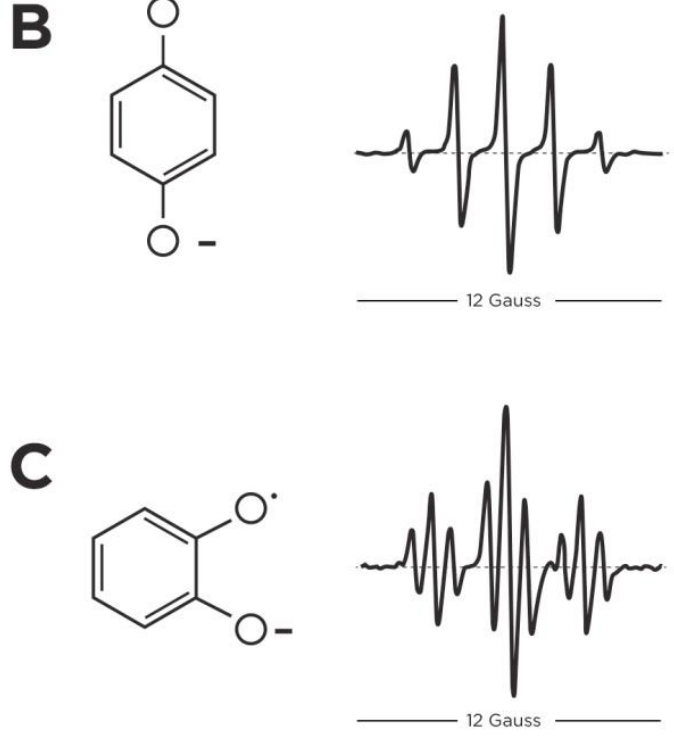

FIGURE 2. A: TEMPOL radical, and ESR Spectrum. B: Hydroquinone (1,4-Dihydroxybenzene) radical anion and ESR spectrum. C: Catechol (1,2-Dihydroxybenzene) radical anion and ESR Spectrum.

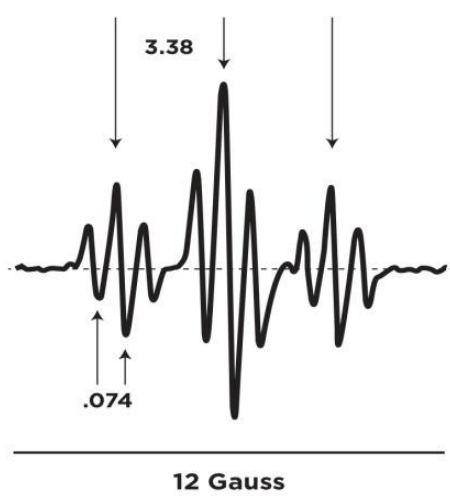

FIGURE 3. ESR spectrum of the catechol radical anion illustrating the two distinct hyperfine splitting constants for the two pairs of equivalent protons. Protons are not explicitly shown.

To clarify, equivalent atoms are in the same environment. The protons next to the oxygen atoms are in the same environment, and are therefore 
equivalent. The remaining two protons are also equivalent. For two equivalent protons, the unpaired electron can "see" both protons as $\operatorname{spin-up~}(\alpha, \alpha)$, one spin-up and one spin-down $(\alpha, \beta)$ or $(\beta, \alpha)$, or both spin-down, $(\beta, \beta)$. Each one of these states corresponds to a different energy; hence, the three lines. The lines are in a 1:2:1 pattern because there are twice as many ways to have the $(\alpha, \beta)$ energy state. In addition, note that there are two distinctly different hyperfine coupling constants for each set of equivalent protons (Fig. 3). This means that one set of equivalent protons has greater electron density than the other set. The two hyperfine splitting constants are 3.38 and 0.74 Gauss. There will also be electron density on the oxygen atoms, but since ${ }^{16} \mathrm{O}$ has a spin quantum number, $\mathbf{I}=0$, there will be no hyperfine splitting from the oxygen atoms. Hückel Molecular Orbital (HMO) calculations show the hyperfine splitting constant of 0.74 can be assigned to the protons next to the oxygen atoms, and the 3.38 hyperfine splitting to the remaining two protons. Reference 2 has a complete description of the benzosemiquinone experiment and HMO calculations.

Turning now to spectrum B (Fig. 2), the same argument that was made for the 1:2:1 pattern seen in the triplet arising from two equivalent protons, can be made for the five line spectrum with the $1: 4: 6: 4: 1$ pattern from four equivalent protons. All protons are spin-up (1 way), one proton is spin-up, and three are spin-down (4 ways), two are spin-up and two are spindown (6 ways), three are spin-up and one is spin down (4 ways), and all four are spin-down (1 way). The hyperfine coupling constant for the hydroquinone radical anion is 2.3 Gauss. As with the catechol radical anion, there is electron density on the oxygen atoms as well, but it cannot be measured with ESR. ${ }^{1}$

Similar compounds with either methyl or t-butyl groups in place of one or more of the protons can also make stable radical anions and can be measured with ESR. These results can also be compared with HMO calculations. The much larger hyperfine splitting seen in TEMPOL means that there is much greater electron density on the nitrogen in TEMPOL than the protons in the SQ radical anions. The results of $\mathrm{HMO}$ calculations also allow one to deduce the spin density of the unpaired electron on the oxygen atoms in the SQ anions, which is much greater than the spin density on the protons.

\footnotetext{
${ }^{1}$ If the SQ radical anions were labeled with ${ }^{17} \mathrm{O}(\mathbf{I}=-5 / 2)$, then the ESR spectra would show hyperfine splitting from the oxygen atoms.
}

\section{MICRO-ESR IN AN INSTRUMENTAL LAB}

The Micro-ESR will also work well as part of an instrumental lab. Most of the important ESR parameters such as RF power, modulation amplitude and phase, gain, sweep rate, spectral width, and resolution can be adjusted by students. The RF power can be arrayed so students can easily set up experiments to learn about and determine power saturation effects. The modulation amplitude and phase can also be adjusted. The effect of these parameters is very important for students to understand. The perylene radical cation is an ideal sample to use to demonstrate the effect of the modulation amplitude. Figure 4 shows the ESR spectrum of the perylene radical cation with the modulation amplitude at 2 Gauss (top) and at 0.02 Gauss (bottom). Much of the fine structure is not visible at full modulation amplitude, but as the value is lowered, the fine structure can be resolved. The modulation phase can also be adjusted. Reference 6 provides a very good explanation of the effects of modulation amplitude and phase.
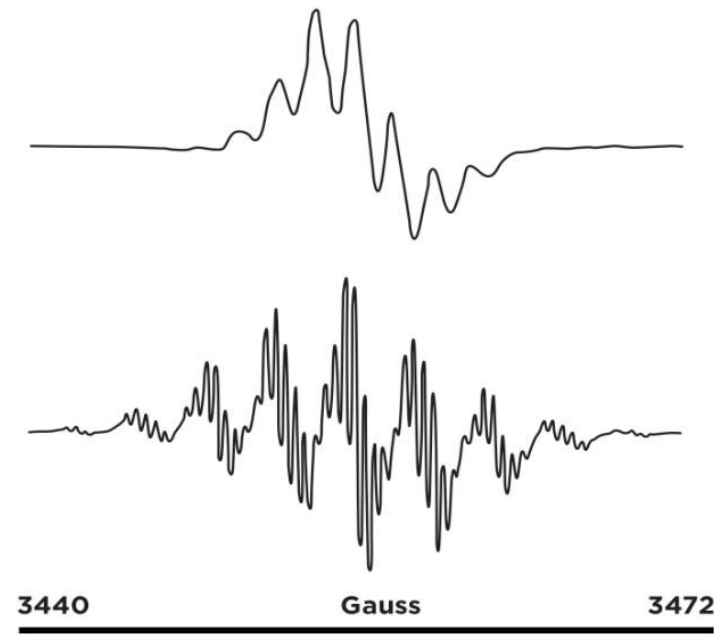

FIGURE 4. Perylene radical cation with the modulation amplitude at 2 Gauss (top), and with modulation amplitude at 0.02 Gauss (bottom)

A wide variety of samples can be used with the Micro-ESR. The spectrometer uses standard $5 \mathrm{~mm}$ quartz ESR tubes, and there is no minimum sample size. Many experiments are run in water or other aqueous solution which requires either $1 \mathrm{~mm}$ ID capillaries or flat cells. We have found that for most lab work, standard melting point capillaries sealed with clay, and inserted into a $5 \mathrm{~mm}$ quartz tube work well. The melting point capillaries are inexpensive, and the quartz ESR tubes can be reused many times. For 
reproducibility, it is important that the capillary is centered in the $5 \mathrm{~mm}$ tube. Centering the tube can be easily achieved with small o-rings, and it is useful for students to test the influence of tube alignment on the resulting spectra. Crystalline solid samples, such as a piece of amethyst crystal small enough to fit in a $5 \mathrm{~mm}$ tube, will give very different spectra as the sample is rotated, but finely powdered samples will not. This is a good example of how important orientation is, and can lead to a good discussion on anisotropy. ${ }^{7}$

Although the students have control over many parameters, the user interface is quite intuitive, and the spectrometer is easy to run and can be operated with a single button if desired. The Micro-ESR is also quite robust. Students cannot damage it by experimenting with parameters, including power. Students do need to be careful not to force tubes into the spectrometer. The bottom plate of the spectrometer can be easily removed if a tube is accidentally dropped in, and students can see what is inside.

The Micro-ESR is provided with off-line processing software, but all data is saved in .csv file format, so is compatible with third party software such as Matlab or Excel. The ease of use, zero maintenance, and versatility of the Micro-ESR make it an excellent choice for undergraduate labs.

\section{REFERENCES}

1. Thiozonyl stable radical anion: $\mathrm{S}_{3}^{-\bullet}$

2. R. Beck and J.W. Nibler, J.Chem.Educ. 66, 263-266 (1989).

3. M. Peric and B. L. Bales, J. Magn. Reson., 169, 27-29 (2004).

4. J. A. Weil and J. R. Bolton, Electron Paramagnetic Resonance: Elementary Theory and Practical Applications, $2^{\text {nd }}$ Ed. (John Wiley \& Sons, Inc., Hoboken, NJ), Section 10.5.

5. J. A. Weil, et al, Appl Magn. Reson., 24, 113-125 (2003).

6. G.R. Eaton, S.S. Eaton, D.P. Barr, and R. T. Weber, Quantitative EPR, (Springewr-Verlag/Wien, Germany, 2010), pp.48-53.

7. Reference 4, Chapters 4 and 5. 